From this equation follows the simplicity of the $G_{M(s)}$ in all except the two pirticular cases $(q, n)=(2,1)$ and $(3,1)$.

The University of Chicago, October 18, 1893.

\title{
NOTE ON MONOGENIC FUNCTIONS OF A SINGLE VARIABLE.
}

By PIOF. T, CRAIG.

THE following remark is so obvious that it seems impossible that it has not been made before; still neither the writer nor those to whom he has spoken have seen it.

Suppose $P(x, y), Q(x, y)$ to be real functions of the real variables $x, y$. Form $P+i Q$ : in order that this shall be a monogenic function of $x+i y=z$ it is necessary first that $P$ and $Q$ be functions satisfying Iaplace's equation

$$
\begin{aligned}
& \nabla P=\frac{\partial^{2} P}{\partial x}+\frac{\partial^{2} P}{\partial y^{2}}=0, \\
& \nabla Q=\frac{\partial^{2} Q}{\partial x^{2}}+\frac{\partial^{2} Q}{\partial y^{2}}=0 .
\end{aligned}
$$

But it is not sufficient that $P$ and $Q$ satisfy this equation. If $P$ be a solution of the equation, $Q$ must be determined by aid of Cauchy's equations

(1)

$$
\frac{\partial P}{\partial x}-\frac{\partial Q}{\partial y}=0
$$

$$
\frac{\partial P}{\partial y}+\frac{\partial Q}{\partial x}=0
$$

that is, $Q$ will be given by the integral

$$
Q=\int_{\left(x_{0}, y_{0}\right)}^{(x, y)}-\frac{\partial P}{\partial y} d x+\frac{\partial P}{\partial x} d y,
$$

in which the condition of integrability is satisfied, since $\nabla P=0$. 
I say, howerer, that a monogenic function of $x+i y$ can be formed by aid of any two solutions of Laplace's equation and without any quadrature. Suppose $P$ and $Q$ to be any two such solutions; they do not then in general satisfy equations (1). If they do satisfy (1), then $P+i Q$ is the function sought. If they do not satisfy (1), write

$$
\begin{aligned}
& Q_{1}=\frac{\partial P}{\partial x}-\frac{\partial Q}{\partial y}, \\
& P_{1}=\frac{\partial P}{\partial y}+\frac{\partial Q}{\partial x} .
\end{aligned}
$$

Then $P_{1}+i Q_{1}$ is a monogenic function of $x+i y$, for

$$
\begin{array}{r}
\frac{\partial P_{1}}{\partial x}-\frac{\partial Q_{1}}{\partial y}=0, \\
\frac{\partial P_{1}}{\partial y}+\frac{\partial Q_{1}}{\partial x}=0, \\
\nabla P_{1}=\nabla Q_{1}=0, \\
\text { since } \nabla P=: \nabla Q=0 .
\end{array}
$$

\section{LAMBERT'S NON-EUCLIDEAN GEOMETRY.}

BY PROF. GEORGE BRUCE HALSTED.

Is the discussion which followed my lecture in the Mathematical Section of the Congress at Chicago, Professor Study of Marburg mentioned that there had recently been brought to light an old paper of Lambert's on what was long after named the non-Enclidean geometry. Professor Klein jotted down on my Lobatschewsky programme the address of Dr. Staeckel, as the person from whom I might hope for definite information; and from his answer to my letter I extract the following highly interesting facts.

This essay of Lambert's bears the title: "Zur Theorie der Parallellinien." It is dated September, 1766, but was first published in 1786 from the papers left by F. Bernoulli, a relative of John Bernoulfi. It appeared in the 